\title{
Reducing Operating Time of a Crawling Robot for Epicardial Surgery
}

\author{
Brina E. Goyette and Cameron N. Riviere \\ The Robotics Institute, Carnegie Mellon University, Pittsburgh, PA
}

\begin{abstract}
HeartLander is a small, mobile robot designed to assist with surgical procedures on the surface of the heart. It crawls within the pericardial sac surrounding the heart. The friction forces HeartLander experiences from the pericardium and the heartbeat reduce locomotion efficiency. We have developed an algorithm that creates a plan for reaching a set of treatment targets, while minimizing the time spent in locomotion. Results from simulation show improvement over a simple greedy technique.
\end{abstract}

\section{INTRODUCTION}

HeartLander is a small robot that has been designed to assist with surgical procedures on the epicardium, or surface of the heart. It is inserted through a small subxiphoid incision, just below the sternum, and placed within the pericardial sac that surrounds the heart. This minimally invasive approach avoids the need to collapse the lung as is required by traditional laparoscopic techniques. By attaching itself to the surface of the heart, HeartLander passively compensates for the motion of the heartbeat. These two advantages mean that the patient can breathe normally during the procedure, and the heart can beat naturally, obviating general anesthesia.

As shown in Fig. 1, HeartLander consists of two body segments, or feet, containing suction chambers that allow them to adhere to the epicardium. Two wires, attached to stepper motors, can push the feet apart or draw them together. By alternating the suction between the front and back feet while adjusting the motors, HeartLander achieves an inchworm-like locomotion. A working channel runs from outside the body to an opening in the front foot, which allows treatments to be administered. Treatments may include, but are not limited to, myocardial injections, inserting pacemaker leads, and ablation of tissue to treat arrhythmias. Many of these interventions involve multiple treatment sites.

HeartLander has two modes of motion. In regular locomotion, HeartLander alternates suction between the front and back feet, and moves the drive wires in and out as necessary to achieve and inchworm-like locomotion. Using this type of locomotion, HeartLander can move from one position on the heart to another. Operating in a fine positioning mode, HeartLander can line the front foot up to a treatment site with increased accuracy. Once HeartLander has moved within one step of the target using regular locomotion, the back foot adheres to the surface. The front foot is left free to be moved by the drive wires. When the correct position is

This work was supported by the NIH under Grant R01 HL078839. reached, instead of pulling the back foot up to the front, the two feet are left apart, and the front foot also adheres to the epicardium, and the treatment can be administered. If there are multiple treatment sites close to one another, HeartLander can move the front foot back towards the back foot, and start the process again without moving the back foot. Keeping the back foot stationary reduces slipping [1].

\section{METHODS}

Due to the heartbeat, friction of pericardial contact, and the presence of pericardial fluid, some slippage occurs during regular locomotion. This decreases HeartLander's effective speed, increasing procedure times. Because the back foot remains fixed during fine positioning, the slippage is reduced. By combining the two modes of movement and maximizing the use of fine positioning, procedure time can be reduced. We first find the smallest set of base points from which HeartLander can use fine positioning to reach all of the treatment sites. We then find the shortest tour from the apex of the heart, where HeartLander is inserted, to each of the base points in the set, and back to the apex for removal. We frame these problems in terms of the Facility Location Problem and the Traveling Salesman Problem respectively, and solve them using $l p \_s o l v e$, a free linear programming solver developed by Michel Berkelarr at Eindhoven University of Technology.

\section{A. Selection of Base Locations}

Selection of the base points from which fine positioning is to be used to reach the treatment sites was based on the Facility Location Problem, which deals with the problem of designating optimal warehouse (or facility) locations for a given set of stores (or sites). There are many possible facility locations, each with an associated building cost and service cost to each individual site. The Facility Location Problem finds the set of facilities that serves each site exactly once with the lowest combination of building and service costs.

The Facility Location Problem can be solved as a Mixed

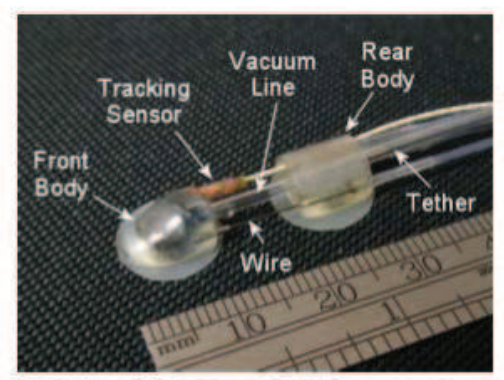

Fig. 1 A view of the HeartLander crawling robot 
Integer Linear Program (MILP). Given a set of variables and a set of constraints, a MILP solver returns the optimal values of the variables. We define variables $F=\left(f_{1}, f_{2}, \ldots, f_{n}\right)$ to represent all possible facilities, and $Y=\left(y_{1}, y_{2}, \ldots, y_{n}\right)$ to be the building costs for each facility. The MILP solver sets $f_{i}=1$ if the facility $i$ is to be created, and $f_{i}=0$ if it is not. We define variable $c_{i j}$ to be the cost for facility $i$ to serve site $j$, and the MILP sets $x_{i j}=1$ if facility $i$ is to serve site $j$, for $i<=n, j<=m$, where $m$ is the number of sites. These variables are used to set up the equations for the MILP as discussed in [2].

In this work, the sites are the treatment sites that HeartLander must reach during a procedure. The potential facility locations are the places on the heart that HeartLander can stop and use fine positioning. Although in reality the selection space is continuous, this algorithm uses a discretization. The fine positioning motion is the method of serving a site. The cost of reaching a site from a given facility is given by an equation based on both the distance that HeartLander must stretch to reach a site $\left(d_{i j}\right)$, and the angle with which it must reach $\left(\theta_{\mathrm{ij}}\right)$. Based on empirical observations of HeartLander's fine positioning, we used the cost function $c_{i j}=7.5 \theta_{\mathrm{ij}}{ }^{2}+2.5 d_{\mathrm{ij}}{ }^{2}$. The angle is weighted more strongly because it has a greater influence on the fine positioning accuracy than the distance. If the angle or distance is out of reach of HeartLander, then $c_{i j}=\infty$, to ensure that the facility is never chosen to serve that site. To reduce the total number of facilities created, the values of $Y$ were set high enough that it is not preferable to create extra facilities. Each facility is given the same cost.

\section{B. Order of Base Locations}

The order or travel from the apex, to each base point, and back to the apex, was determined by solving the Traveling Salesman Problem. This problem looks to find the optimal tour from a traveling salesman's home city, to each city in which he has business, and back. The cost of the tour could be travel time, distance, or price. It too can be solved as a MILP. For a set of $n$ cities, we define $c_{i j}$ to be the cost of traveling between city $i$ and city $j$, and $x_{i j}$ to be a variable that the MILP solver sets to 1 if the salesman travels from city $i$ to city $j$ in his tour, and 0 otherwise. The tour is constrained to be one
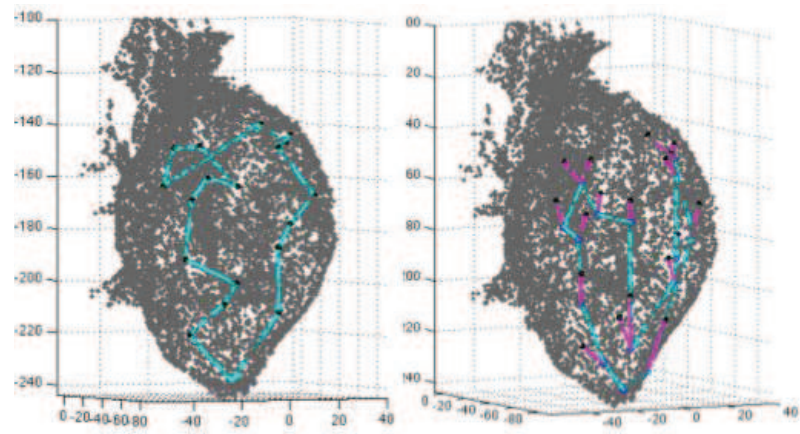

Fig. 2 Tours of random treatment sites on the heart. Left: Tour from the greedy approach. Right: Tour from the MILP approach. Grey dots show potential base locations. Black dots show the treatment sites. Cyan lines show regular locomotion paths, and magenta lines show fine positioning paths. continuous loop. The MILP equations are then set up as discussed in [3].

In our algorithm, the traveling salesman is HeartLander, and the "cities" it must visit are the base locations for the fine positioning, as determined by the Facility Location Problem. The cost of moving between two locations is based on the distance HeartLander must travel. However, it is much more time consuming for HeartLander to move horizontally than vertically (toward the patient's head). For this reason, horizontal movement is weighted more heavily than vertical movement in our cost function, giving us $c_{i j}=d_{i j}+10 h_{i j}$, where $d_{i j}$ is the distance between cities $i$ and $j$, and $h_{i j}$ is the horizontal component of the distance between cities $i$ and $j$.

\section{RESULTS}

We evaluated our algorithm against a simple greedy approach. The greedy approach was not able to take fine positioning into consideration, and did not use weighted distances. It simply chose the closest site to the previously chosen site, until all sites had been visited, starting and ending with the apex.

Initially, a random set of treatment sites was defined on the surface of the heart (Fig. 2). Such a pattern is not typical of a real surgical procedure, so two other patterns also were defined. The first simulated treating the perimeter of a region of damaged tissue, while the second simulated treating the entire area of the same damaged region. The results are reported in Table I. The MILP approach achieved a decrease in cost in each case, generally by minimizing costly horizontal motion.

TABLE I

RESURTS OF EVALUATION

\begin{tabular}{l|r|r|r|} 
& Random & Perimeter & \multicolumn{1}{c|}{ Area } \\
\hline $\begin{array}{l}\text { Number of Treatment Sites } \\
\text { Number of Facilities }\end{array}$ & 18 & 31 & 30 \\
\hline $\begin{array}{l}\text { Distance of Plan Tour } \\
\text { Distance of Greedy Tour }\end{array}$ & 415 units & 242 units & 233 units \\
\hline $\begin{array}{l}\text { Decrease in Distance } \\
\text { Cost of Plan Tour }\end{array}$ & 419 units & 270 units & 327 units \\
\hline Cost of Greedy Tour & $170 \%$ & $10.4 \%$ & $28.7 \%$ \\
\hline Decrease in Cost & 3283 units & 1259 units & 1386 units \\
\hline & $45.7 \%$ & 1412 units & 2046 units \\
\hline
\end{tabular}

\section{DISCUSSION}

By optimizing the treatment plan using a combination of the Facility Location Problem and the Traveling Salesman Problem, we obtained shorter treatment plans than with a simple greedy approach. Decreasing procedure times can be expected to produce decreased operating room costs. Future work will involve experimental verification.

\section{REFERENCES}

[1] N. Patronik, T. Ota, M. Zenati, and C. Riviere, "A miniature mobile robot for navigation and positioning on the beating heart," IEEE Trans. Robot., vol. 25, pp. 1109-1124, 2009.

[2] R.Z. Farahani and M. Hekmatfar, Facility Location, Springer, 2009

[3] F. Rossi, P.V. Beek, and T. Walsh, Handbook of Constraint Programming, Elsevier, 2006. 